\title{
Computations of generalized Dolbeault cohomology
}

\author{
Gil R. Cavalcanti ${ }^{1}$ \\ Utrecht University
}

\begin{abstract}
We study the (generalized Dolbeault) cohomology of generalized complex manifolds in 4 real dimensions. We show that in 4 real dimensions, the first cohomology around a nondegenerate type change point is given by holomorphic $(1,0)$ forms defined on the type change locus. We use this to compute the cohomology of a neighbourhood of a compact component of the type change locus as well as that of the blow-up of a type change point. Finally, we use these computations to determine the generalized cohomology of some concrete examples.
\end{abstract}

Keywords: Generalized complex structures, Dolbeault cohomology.

PACS: MSC2000 numbers:53C15, 32Q55, 53D35.

\section{INTRODUCTION}

Generalized complex structures, introduced by Hitchin [8] and further developed by Gualtieri [7], provide a simultaneous generalization of complex and symplectic structures, are related to Poisson and Dirac geometry [7, 10, 5] and have applications to string theory as they are a vital ingredient in general solution of the $(2,2)$ supersymmetric $\sigma$-model [6, 11].

Geometrically, generalized complex structures share several properties familiar from complex and symplectic geometry. Particularly relevant for these notes is that a generalized complex structure induces a decomposition of the space of forms similar to the $(p, q)$ decomposition on a complex manifold and, further, this decomposition provides a decomposition of the exterior derivative into operators analogous to $\partial$ and $\bar{\partial}$ [7]. Therefore there is an analogue of Dolbeault cohomology for generalized complex manifolds which agrees with Dolbeault cohomology of complex manifolds and is isomorphic to ordinary cohomology of symplectic manifolds [1].

While symplectic and complex manifolds provide examples of generalized complex manifolds, these are just two ends on an spectrum of generalized complex structures: pointwise, generalized complex structures are distinguished by their type with symplectic being type zero and complex corresponding to the maximal type. Furthermore, in a manifold, the type does not need to be constant and can jump up at submanifolds. This feature of type change was used in [2] and [3] to produce generalized complex structures on $(2 n+1) \mathbb{C} P^{2} \# m \overline{\mathbb{C} P^{2}}$, manifolds which do not admit complex or symplectic structures for $n>1$.

In these notes we show how to compute the generalized Dolbeault cohomology of

1 g.r.cavalcanti@uu.nl 
these manifolds and prove that it concentrates in middle dimension. As a consequence, we see that these manifolds satisfy the $\partial \bar{\partial}$-lemma. This is done by computing the cohomology around components of the type change locus as well as the effect of generalized complex blow-up to cohomology. These computations can be used to study the cohomology of other type changing generalized complex structures.

\section{GENERALIZED COMPLEX STRUCTURES}

In this section we recall the definition, basic properties and examples of generalized complex structures, following [7]. We start with the linear algebra of a generalized complex structure.

\subsection{Linear algebra}

For any vector space $V$, the sum $V \oplus V^{*}$ has a natural symmetric pairing

$$
\langle X+\xi, Y+\eta\rangle=\frac{\eta(X)+\xi(Y)}{2} .
$$

A (linear) generalized complex structure on $V$ is a linear complex structure $\mathscr{J}$ on $V \oplus V^{*}$ orthogonal with respect to the natural pairing. A linear generalized complex structure, $\mathscr{J}$, splits the complexification $V_{\mathbb{C}} \oplus V_{\mathbb{C}}^{*}$ into its $\pm i$-eigenspaces. The $+i$-eigenspace of $\mathscr{J}$ is a maximal isotropic subspace $L \subset V_{\mathbb{C}} \oplus V_{\mathbb{C}}^{*}$ satisfying

$$
L \cap \bar{L}=\{0\} .
$$

Given any such subspace, one can define a generalized complex structure by declaring it to be linear, equal to $+i$ on $L$ and $-i$ on $\bar{L}$. Hence generalized complex structures on $V$ are equivalent to maximal isotropic subspaces $L \subset V_{\mathbb{C}} \oplus V_{\mathbb{C}}^{*}$ satisfying (1.1).

Another description of generalized complex structures comes from the space of spinors for $V \oplus V^{*}$. The space of forms $\wedge^{\bullet} V^{*}$ is naturally the space of spinors for $V \oplus V^{*}$, since the action of $X+\xi \in V \oplus V^{*}$ on a form $\rho$ given by

$$
(X+\xi) \cdot \rho=i_{X} \rho+\xi \wedge \rho
$$

extends to an action of the Clifford algebra of $V \oplus V^{*}$ on $\wedge^{\bullet} V^{*}$. Observe that given a form $\rho \in \wedge^{\bullet} V_{\mathbb{C}}^{*}$, the subspace

$$
L_{\rho}:=\left\{v \in V_{\mathbb{C}} \oplus V_{\mathbb{C}}^{*}: v \cdot \rho=0\right\} \subset V_{\mathbb{C}} \oplus V_{\mathbb{C}}^{*}
$$

is always an isotropic subspace which depends only on the line generated by $\rho$ and not $\rho$ itself. The condition that $L_{\rho}$ is maximal isotropic translates into algebraic properties of $\rho$, namely $\rho$ must be of the form (see [4])

$$
\rho=e^{B+i \omega} \wedge \Omega
$$


where $B, \omega \in \wedge^{2} V^{*}$ and $\Omega$ is a decomposable complex form.

Definition 1. Forms of the algebraic type (1.2) are called pure.

The condition $L \cap \bar{L}=\{0\}$ can also be expressed in terms of $\rho=e^{B+i \omega} \wedge \Omega$. Indeed, this condition holds if and only if (see [4])

$$
0 \neq(\rho, \bar{\rho})=\Omega \wedge \bar{\Omega} \wedge(2 i \omega)^{n-k},
$$

where $k=\operatorname{deg}(\Omega), \operatorname{dim}(V)=2 n$. and $(\cdot, \cdot)$ denotes the natural spin invariant pairing on spinors, also known as the Chevalley pairing.

Definition 2. Given a generalized complex structure $\mathscr{J}$ on $V$, the line $K \subset \wedge^{\bullet} V_{\mathbb{C}}^{*}$ annihilating the $+i$-eigenspace of $\mathscr{J}$ is the canonical line. If $e^{B+i \omega} \wedge \Omega$ is one generator for that line, the degree of $\Omega$ is the type of $\mathscr{J}$ and the parity of $\Omega$ is the parity of $\mathscr{J}$.

A generalized complex structure $\mathscr{J}$ on $V$ determines a decomposition of $\wedge V_{\mathbb{C}}^{*}$ into the eigenspaces of the Lie algebra action of $\mathscr{J}$. These spaces can be concretely described as follows (see [7])

$$
U^{k}=\wedge^{n-k} \bar{L} \cdot K \quad-n \leq k \leq n,
$$

where $K$ is the canonical line and $\cdot$ denotes the Clifford action of $\wedge^{\bullet} \bar{L} \subset \operatorname{Clif}\left(V_{\mathbb{C}} \oplus V_{\mathbb{C}}^{*}\right)$.

Example 3. A complex structure $I$ on a vector space $V$ induces a generalized complex structure $\mathscr{J}_{I}$ on $V$ defined by the following matrix in the natural splitting $V \oplus V^{*}$

$$
\mathscr{J}_{I}=\left(\begin{array}{cc}
-I & 0 \\
0 & I^{*}
\end{array}\right) .
$$

For this structure we see that $L=V^{0,1} \oplus V^{* 1,0}$ and the canonical line is $K=\wedge^{n, 0} V^{*}$, where $\operatorname{dim}(V)=2 n$.

In this case one can easily see that the spaces $U^{k}$ decomposing the space of forms $\wedge^{\bullet} V_{\mathbb{C}}^{*}$ are given by $U^{k}=\oplus_{p-q=k} \wedge^{p, q} V^{*}$, where $\wedge^{p, q} V^{*}$ is the standard $(p, q)$ decomposition of forms on a complex space.

Example 4. A symplectic structure $\omega$ on a vector space $V$ can be seen as an invertible map $\omega: V \longrightarrow V^{*}$ and hence also induces a generalized complex structure $\mathscr{J}_{\omega}$ on $V$ defined by the following matrix in the natural splitting $V \oplus V^{*}$

$$
\mathscr{J}_{\omega}=\left(\begin{array}{cc}
0 & -\omega^{-1} \\
\omega & 0^{*}
\end{array}\right) \text {. }
$$

For this structure we see that $L=\left\{X-i \omega(X): X \in V_{\mathbb{C}}\right\}$ and the canonical line $K$ is generated by the form $e^{i \omega}$.

The spaces $U^{k}$ decomposing the space of forms $\wedge^{\bullet} V_{\mathbb{C}}^{*}$ are given by (see [1])

$$
U^{k}=e^{i \omega} e^{\frac{-\omega^{-1}}{2 i}} \wedge^{n-k} V_{\mathbb{C}}^{*}
$$


where $\omega^{-1}$ is regarded as a bivector and acts on forms by interior product, decreasing the degree by 2 . So, in the symplectic case, the $U^{k}$ decomposition is different from the decomposition according to degree, but these are isomorphic.

Example 5. A 2-form $B \in \wedge^{2} V^{*}$ can be seen as a map $B: V \longrightarrow V^{*}$ and gives rise to an orthogonal map of $V \oplus V^{*}$, called a $B$-field transform, given by

$$
e^{B}=\left(\begin{array}{cc}
\mathrm{Id} & 0 \\
B & \mathrm{Id}^{*}
\end{array}\right) .
$$

Therefore 2-forms act on generalized complex structures: given a generalized complex structure $\mathscr{J}$ and a 2-form $B$, the automorphism $\mathscr{J}^{B}=e^{B} \mathscr{J} e^{-B}$ is another generalized complex structure. If $\mathscr{J}$ has canonical line $K$, then $\mathscr{J}^{B}$ has canonical line $e^{B} \wedge K$ and similarly, the decomposition of forms for $\mathscr{J}^{B}$ is given by $\left(U_{\mathscr{J}^{B}}\right)^{k}=e^{B} \wedge U_{\mathscr{J}}^{k}$. In particular, for a structure obtained by a $B$-field transform of a symplectic structure $\omega$ the canonical bundle is given by $e^{B+i \omega}$ and

$$
U^{k}=e^{B+i \omega} e^{-\omega^{-1}} \wedge^{n-k} V_{\mathbb{C}}^{*}
$$

\subsection{Integrability and cohomology}

An almost generalized complex structure on a manifold $M^{2 n}$ is a smooth assignment of a generalized complex structure $\mathscr{J}$ to each tangent space $T_{x} M$. Integrability of this structure is a requirement of compatibility between the differential structure of $M$ and $\mathscr{J}$. This requirement also depends on a pre-chosen real closed 3-form $H$ on $M$ which is used to form the differential operator $d_{H}=d+H \wedge$.

To state this precisely, recall that $\mathscr{J}$ splits the space $\wedge^{\bullet} T_{\mathbb{C}}^{*} M$ into subspaces $U^{k}$, as in (1.4). If we denote by $\mathscr{U}^{k}$ the space of (local) sections of $U^{k}$, we say that $\mathscr{J}$ is integrable with respect to to $H$, or if $H$ is clear from the context, simply that $\mathscr{J}$ is integrable if

$$
d_{H}: \mathscr{U}^{n} \longrightarrow \mathscr{U}^{n-1}
$$

The condition above is equivalent to requiring the existence of $X+\xi \in C^{\infty}\left(T_{\mathbb{C}} M \oplus T_{\mathbb{C}}^{*} M\right)$ such that

$$
d_{H} \rho=(X+\xi) \cdot \rho \quad \text { for all } \rho \in \mathscr{U}^{n} .
$$

Whenever $\mathscr{J}$ is integrable, one also has that (see [7])

$$
d_{H}: \mathscr{U}^{k} \longrightarrow \mathscr{U}^{k+1} \oplus \mathscr{U}^{k-1},
$$

which allows us to define operators

$$
\partial_{\mathscr{J}}: \mathscr{U}^{k} \longrightarrow \mathscr{U}^{k+1} \quad \text { and } \quad \bar{\partial}_{\mathscr{J}}: \mathscr{U}^{k} \longrightarrow \mathscr{U}^{k-1}
$$


as the projections of $d_{H}$ into the relevant space. If there is no danger of confusion, e.g., when there is only one generalized complex structure in consideration, we denote these operators simply by $\partial$ and $\bar{\partial}$. From $d_{H}^{2}=0$, we get that

$$
\partial^{2}=\bar{\partial}^{2}=\partial \bar{\partial}+\bar{\partial} \partial=0
$$

Therefore we can define the generalized (Dolbeault) cohomology of a generalized complex manifold as the cohomology of the operator $\bar{\partial}$.

In [7], Gualtieri proved that generalized cohomology of a compact generalized complex manifold $M^{2 n}$ is finite dimensional. In [1], the author proved that for $M$ compact, the Mukai pairing gives rise to a nondegenerate pairing between $G H^{k}(M)$ and $G H^{-k}(M)$ and hence these spaces have the same dimension. Further, the Euler characteristic can be computed directly from $G H(M)$ :

$$
\chi(M)=(-1)^{n+\operatorname{par}(\mathscr{J})}\left(\sum_{i=-n}^{n}(-1)^{i} g b_{i}\right),
$$

where $\operatorname{par}(\mathscr{J})$ is the parity of $\mathscr{J}$ and $g b_{i}=\operatorname{dim}\left(G H^{i}(M)\right)$ are the generalized Betti numbers.

Example 6. If $M$ is endowed with an almost complex structure $I$, it has an almost generalized complex structure induced by $I, \mathscr{J}_{I}$, as in Example 1.1. Taking $H=0$ to be the underlying 3-form, integrability of the generalized complex structure means that $d: \Omega^{n, 0}(M) \longrightarrow \Omega^{n, 1}(M)$, which implies integrability of $I$ as a complex structure. In this case, one easily sees that the decomposition $d=\partial+\bar{\partial}$ is nothing but the usual one for complex manifolds. In this case the generalized cohomology agrees with Dolbeault cohomology.

Example 7. If $M$ is endowed with a nondegenerate 2-form $\omega$, it has an almost generalized complex structure induced by $\omega, \mathscr{J}_{\omega}$, as in Example 1.1. Taking $H=0$ to be the underlying 3-form, integrability of the generalized complex structure means that $d \omega=0$ and hence $M$ is a symplectic manifold. Further, $\mathscr{U}^{k}=e^{i \omega} e^{\frac{-\omega}{2 i}} \Omega^{n-k}(M)$ and one can show that $\partial$ and $\bar{\partial}$ are given by (see [1])

$$
\begin{aligned}
& \bar{\partial}\left(e^{i \omega} e^{\frac{-\omega}{2 i}} \alpha\right)=e^{i \omega} e^{\frac{-\omega}{2 i}} d \alpha \\
& \partial\left(e^{i \omega} e^{\frac{-\omega}{2 i}} \alpha\right)=\frac{1}{2 i} e^{i \omega} e^{\frac{-\omega}{2 i}} d^{\mathscr{J} \omega} \alpha,
\end{aligned}
$$

where $d^{\mathscr{J}} \omega=-i(\partial-\bar{\partial})$ and in this case it is also given by $d^{\mathscr{J}_{\omega}}=\left[d, \omega^{-1}\right]$, which is the symplectic adjoint of $d$, as introduced in [9].

Observe that, due to equation $(1.6)$, in this case the generalized cohomology is isomorphic to the usual cohomology of the manifold.

Example 8. Given $B \in \Omega^{2}(M)$, we can use it to produce the $B$-field transform of $T M \oplus T^{*} M$. Since this is an orthogonal tranformation of $T M \oplus T^{*} M$, in order for it to map generalized complex structures to generalized complex structures one only needs 
that the corresponding map on pure forms, i.e, $e^{B} \wedge$, commutes with $d_{H}$. This means that we need $\left[d_{H}, e^{B}\right]=0$, but one can easily compute

$$
\left[d_{H}, e^{B}\right]=d_{H} e^{B}-e^{B} d_{H}=e^{B}\left(d B+d_{H}\right)-e^{B} d_{H}=e^{B} d B
$$

showing that this map will preserve integrability independently of the structure in consideration if and only if $B$ is closed.

Diffeomorphisms and closed $B$ forms form the symmetry group of $T M \oplus T^{*} M$ hence given a generalized complex structure $\mathscr{J}$ we consider any other generalized complex structure obtained from $\mathscr{J}$ by diffeomorphisms and $B$-field transforms equivalent to $\mathscr{J}$.

Example 9. A generalized complex structure does not necessarily have constant type. For example, still with $H=0$, consider the following form defined on $\mathbb{C}^{2}$ :

$$
\rho=z_{1}+d z_{1} d z_{2} \text {. }
$$

Pointwise, this form has the right algebraic type, satisfies $(\rho, \bar{\rho}) \neq 0$, hence it defines an almost generalized complex structure $\mathscr{J}$. Further

$$
d \rho=z_{1}=-\partial_{z_{2}} \cdot \rho
$$

showing that $\mathscr{J}$ is integrable.

If $z_{1} \neq 0, \mathscr{J}$ is determined by the line generated by $e^{\frac{d z_{1} d z_{2}}{z_{1}}}$, showing that it is a $B$-field transform of a symplectic structure (hence type 0 ). For $z_{1}=0$, the canonical bundle is given by $d z_{1} \wedge d z_{2}$, showing that $\mathscr{J}$ is of complex type (type 2 ).

Example 10. For an example with compact type change locus, one can observe that this structure is invariant under translations in the $z_{2}$ coordinate. This allows us to take a quotient of $\mathbb{C}^{2}$ by a $\mathbb{Z}^{2}$ action to obtain a type changing generalized complex structure on $\mathbb{C} \times T^{2}$, where the type change is the elliptic curve over $z_{1}=0$.

In 4-dimensions, any nondegenerate type change point has a neighbourhood where the generalized complex structure is equivalent to the one given in Example 1.2 [3]. This makes that example particularly interesting as it tells us that studying particular features of that generalized complex structure has applications to generalized complex structures on other spaces.

\section{COHOMOLOGY COMPUTATIONS}

Now we start with our cohomology computations. We start off with the generalized cohomology around a nondegenerate type change point. With that basic result at hand, we can compute the cohomology of a neighbourhood of a type change torus (which in a sense is the generic compact type change locus) and effects of blow-up to the generalized cohomology of a 4-manifold.

The first result is known since Gualtier's thesis [7], but to my knowledge no reference can be found for it. Since we will need it in the sequence, I will commit it to paper. 
Lemma 2.1. If $M^{2 n}$ has a generalized complex structure which is generically symplectic, but whose type is not constant, then $G H^{n}(M)=\{0\}$

Proof. Indeed, any $\bar{\partial}$-closed section of the canonical bundle is also $d_{H}$-closed, since $\partial: \mathscr{U}^{n} \longrightarrow \mathscr{U}^{n+1}$ vanishes trivially and $d_{H}=\partial+\bar{\partial}$. In the symplectic locus the canonical bundle is generated by $e^{B+i \omega}$, hence, in this locus, sections of the canonical bundle are given by $f e^{B+i \omega}$ and these are $d_{H}$-closed if and only if $f$ is constant. However, the degree zero part of any section of the canonical bundle defined at the type change locus must vanish, hence the form $e^{B+i \omega}$ is not well defined in the type change locus and the only globally defined $\bar{\partial}$-closed form on $\mathscr{U}^{n}$ is the trivial one.

Now we study $G H^{1}(M)$.

Proposition 2.2. Let $M$ be $\mathbb{C}^{2}$ endowed with the generalized complex structure of Example 1.2 Then $G H^{1}(M)$ is given by holomorphic $(1,0)$-forms defined on $\left[z_{1}=0\right]$.

Proof. Since $\mathbb{C}^{2}$ is naturally endowed with a complex structure, we will use symbols $\partial$ and $\bar{\partial}$ to refer to the usual operators in $\mathbb{C}^{2}$ which go by these names and will use $\partial \mathscr{f}$ and $\bar{\partial}_{\mathscr{J}}$ to refer to the operators induced by $\mathscr{J}$.

We start characterizing sections of $\mathscr{U}^{2}$. Since $\mathscr{J}$ is symplectic away from $\left[z_{1}=0\right]$, we see that the decomposition of forms and the $\bar{\partial}$ operator are given by the $B$-field transform of equations (1.4) and (1.6). So, for example, the generic element in $\mathscr{U}^{2}$, away from $\left[z_{1}=0\right]$, is of the form

$$
e^{B+i \omega} e^{\frac{-\omega^{-1}}{2 i}} f=\left(1+\frac{d z_{1} d z_{2}}{z_{1}}\right) f,
$$

with $f \in \Omega^{0}\left(\mathbb{C}^{2} \backslash\left[z_{1}=0\right]\right)$. This defines an element in $\mathscr{U}^{2}\left(\mathbb{C}^{2}\right)$ if and only if it extends smoothly to the locus $\left[z_{1}=0\right]$, which means that $f \in \Omega^{0}\left(\mathbb{C}^{2}\right)$ and it must be of the form $f=z_{1} g$, for some smooth function $g$.

Now we move on to determine the forms in $\mathscr{U}^{1}$. As before, the generic element in $\mathscr{U}^{1}$ is given, away from $\left[z_{1}=0\right]$, by

$$
e^{B+i \omega} e^{\frac{-\omega^{-1}}{2 i}} \alpha=\left(1+\frac{d z_{1} d z_{2}}{z_{1}}\right) \alpha
$$

with $\alpha \in \Omega^{1}\left(\mathbb{C}^{2} \backslash\left[z_{1}=0\right]\right)$. And this is an element in $\mathscr{U}^{1}\left(\mathbb{C}^{2}\right)$ if and only if we can extend $\alpha$ so that the right hand side is a well defined form. From the degree 1 part, we see that $\alpha$ must extend smoothly and, from the degree 3 part, we see that $\alpha^{0,1}$, the $(0,1)$ component of $\alpha$, must be of the form

$$
\alpha^{0,1}=z_{1} \beta, \quad \text { with } \beta \in \Omega^{0,1}\left(\mathbb{C}^{2}\right) .
$$

Now we determine the $\bar{\partial}_{\mathscr{J}}$-closed forms in $\mathscr{U}^{1}(M)$. Firstly, for $\alpha$ as above,

$$
\bar{\partial}_{\mathscr{J}}\left(e^{B+i \omega} e^{\frac{-\omega^{-1}}{2 i}} \alpha\right)=\left(e^{B+i \omega} e^{\frac{-\omega^{-1}}{2 i}} d \alpha\right)
$$


and, checking the components of different degrees in the expression above, one sees that $\alpha$ must be $d$-closed for the expression above to vanish. So, the $\bar{\partial} \mathscr{J}$-closed forms in $\mathscr{U}^{1}$ are in bijection with $d$-closed forms $\alpha \in \Omega^{1}\left(\mathbb{C}^{2}\right)$ for which 2.1$)$ holds.

From $(2.1)$, we see that $\left.\bar{\partial} \alpha\right|_{\left[z_{1}=0\right]}=0$, hence $a\left(z_{2}\right)=\left.\alpha\right|_{\left[z_{1}=0\right]}$ is a holomorphic $(1,0)$ form defined on the type change locus and, denoting by $\pi$ the projection onto the second coordinate, $\tilde{\alpha}=\alpha-\pi^{*} a$ is a closed form satisfying (2.1) (hence determines a closed form on $\mathscr{U}^{1}\left(\mathbb{C}^{2}\right)$ ) and whose restrictionto the type change locus vanishes.

Now we prove that $\tilde{\alpha}$ represents the trivial cohomology class in $G H^{1}\left(\mathbb{C}^{2}\right)$. Since $H^{1}\left(\mathbb{C}^{2}\right)=\{0\}, \tilde{\alpha}=d f$, for some $f \in \Omega^{0}\left(\mathbb{C}^{2}\right)$ and we can choose $f$ so that $f(0,0)=0$, which implies that $f$ vanishes along the type change locus. Then the element we are considering is

$$
e^{B+i \omega} e^{\frac{-\omega^{-1}}{2 i}} \tilde{\alpha}=d f+\frac{d z_{1} \wedge d z_{2}}{z_{1}} \bar{\partial} f
$$

subject to 2.1$)$, i.e.,

$$
\bar{\partial} f=z_{1} \beta \quad \text { for some smooth } \beta .
$$

Applying $\bar{\partial}$ to $(2.2)$, we see that $\bar{\partial} \beta=0$ and hence $\beta=\bar{\partial} \varphi$, for some $\varphi \in \Omega^{0}\left(\mathbb{C}^{2}\right)$, so $\bar{\partial} f=\bar{\partial}\left(z_{1} \varphi\right)$ and $\tilde{f}=f-z_{1} \varphi$ is a holomorphic function which vanishes along $\left[z_{1}=0\right]$, hence it must be of the form $z_{1} \tilde{\varphi}$, for some holomorphic function $\tilde{\varphi}$. Therefore we have $f=z_{1}(\varphi+\tilde{\varphi})$. According to the first part of this proof, the function $z_{1}(\varphi+\tilde{\varphi})$ gives rise to a well defined element in $\mathscr{U}^{2}(M)$ namely, $\left(1+\frac{d z_{1} \wedge d z_{2}}{z_{1}}\right) z_{1}(\varphi+\tilde{\varphi})$ and

$$
\bar{\partial}_{\mathscr{J}}\left(1+\frac{d z_{1} \wedge d z_{2}}{z_{1}}\right) z_{1}(\varphi+\tilde{\varphi})=\left(1+\frac{d z_{1} \wedge d z_{2}}{z_{1}}\right) d f=\left(1+\frac{d z_{1} \wedge d z_{2}}{z_{1}}\right) \tilde{\alpha}
$$

so the $\bar{\partial}_{\mathscr{J}}$-closed form determined by $\tilde{\alpha}$ represents the trivial $\bar{\partial} \mathscr{J}$ cohomology class and the class determined by $\alpha$ is the same as

$$
\left(1+\frac{d z_{1} \wedge d z_{2}}{z_{1}}\right) a\left(z_{2}\right)=a\left(z_{2}\right)
$$

Conversely, one can easily see that any nontrivial holomorphic $(1,0)$-form on $z_{2}$ determines a nontrivial cohomology class.

According to [2], any compact component of the type change locus containing only nondegenerate points is a torus, which itself inherits a complex structure making it into a smooth elliptic curve. The argument used above can be adapted to compute the generalized cohomology of a neighbourhood of such torus.

Theorem 2.3. Let $M$ be a 2-disc bundle over a 2-torus with a generalized complex structure which is symplectic away from the zero section, $T$, and which has nondegenerate type change along $T$. Then $G H^{2}(M)=\{0\}$ and $G H^{1}(M)=\mathbb{C}$, generated by the holomorphic $(1,0)$ differential in the elliptic curve $T$.

Proof. Due to Lemma 2.1, we only need to prove that $G H^{1}(M)=\mathbb{C}$.

Since all the points in the type change locus are nondegenerate, according to [3] around each point in the type change locus we can find a $B$-field and a diffeomorphism 
which renders the structure equal to the structure from Example 1.2. Observe however that now the complex structure is not canonical but depends on our choices $(B$-field and diffeomorphism). So we find a good cover for a neighbourhood of the zero section by open sets $V_{i}$ so that in each set the structure is $B$-diffeomorphic to the standard one.

As before, we consider first the symplectic locus, where elements of $\mathscr{U}^{1}$ are given by forms of the form

$$
e^{B+i \omega} \alpha \quad \alpha \in \Omega^{1}(M \backslash T ; \mathbb{C}) .
$$

In order for this to be a globally defined element of $\mathscr{U}^{1}$ we need that $\alpha$ is smooth and that, on $V_{i}$,

$$
\alpha^{0,1}=z_{1} \beta_{i} \quad \text { for some } \beta_{i} \in \Omega^{0,1}\left(V_{i}\right) .
$$

The same argument used in Proposition 2.2 shows that for 2.3 to be $\partial \mathscr{g}$-closed, one must have $d \alpha=0$ and restricting $\alpha$ to the zero section, we see that $a:=\left.\alpha\right|_{T}$ is a holomorphic $(1,0)$ form on $T$. Letting $\pi: M \longrightarrow T$ be the projection, $\tilde{\alpha}=\alpha-\pi^{*} a$ is a closed form which restricts to zero on the zero section, therefore it is exact, say $\tilde{\alpha}=d f$, and we can choose $f$ so that it vanishes at a point (and hence everywhere) in $T$.

From the proof of Proposition 2.2 we see that on each $V_{i}, f=z_{1} \varphi_{i}$, for some function $\varphi_{i}$, showing that $e^{B+i \omega} f$ is a well defined section of $\mathscr{U}^{2}$ and that

$$
\bar{\partial}_{\mathscr{J}}\left(e^{B+i \omega} f\right)=e^{B+i \omega} \tilde{\alpha} .
$$

Hence $(2.3)$ is in the same $\bar{\partial}_{\mathscr{J}}$-cohomology class as

$$
e^{B+i \omega} a
$$

which is a nontrivial class, as it is nontrivial even locally. This shows that $G H^{1}(M)$ is isomorphic to the space of holomorphic $(1,0)$ differentials on the type change locus. Since the type change locus in this case is an elliptic curve, we have that $G H^{1}(M)=$ $\mathbb{C}$.

\section{BLOW-UP}

In joint work with Gualtieri the author introduced the generalized complex blow-up in [3]. In a nutshell, the idea is simply to take a nondegenerate type change point, which therefore has a neighbourhood in which the generalized complex structure is equivalent to the one in Example 1.2 and then do the complex blow up of the origin in $\mathbb{C}^{2}$. In this setting, the pull back of the canonical bundle to the blow up $\widetilde{\mathbb{C}^{2}}$ determines a generalized complex structure on that space. Concretely, $\mathbb{C}^{2}$ is endowed with generalized complex structure

$$
z_{1}+d z_{1} \wedge z_{2}
$$


and $\widetilde{\mathbb{C}^{2}}$ is parametrized by two coordinate charts $\left(u_{1}, u_{2}\right)$ and $\left(v_{1}, v_{2}\right)$ and the blow down map is given in each of these charts by

$$
\begin{array}{lll}
z_{1}=u_{1} & z_{2}=u_{1} u_{2} & \text { for } u_{1} \neq 0 \\
z_{1}=v_{1} v_{2} & z_{2}=v_{2} & \text { for } v_{2} \neq 0 .
\end{array}
$$

Hence the pull back of the canonical bundle of the structure on $\mathbb{C}^{2}$ is a multiple of

$$
1+d u_{1} \wedge d u_{2} \quad \text { and } \quad v_{1}+d v_{1} \wedge d v_{2}
$$

Observe that, by construction $\pi^{*} U^{2}=U^{2}$, where $\pi: \widetilde{\mathbb{C}^{2}} \longrightarrow \mathbb{C}^{2}$ is the blow-down map. Therefore we also have $\pi^{*}\left(U^{k}\right)=U^{k}$ and hence $\pi^{*}$ gives rise to a map of generalized cohomologies.

Lemma 3.1. In the situation above $G H^{1}\left(\widetilde{\mathbb{C}^{2}}\right)=\pi^{*} G H^{1}\left(\mathbb{C}^{2}\right)$.

Proof. $\widetilde{\mathbb{C}^{2}}$ is covered by two open sets, $U$ and $V$, the $u$ and $v$ coordinate charts. On $U$ the structure is purely symplectic, and due to $(1.6)$, the generalized cohomology of that set is equivalent to the cohomology of $\mathbb{C}^{2}$, i.e., it is trivial. On $V$, the structure is the same as in Example 1.2, hence, according to Proposition 2.2. $G H^{1}(V)$ is given by the holomorphic $(1,0)$ differentials along the type change locus.

Now, Mayer-Vietoris tells us that $G H^{1}\left(\widetilde{\mathbb{C}^{2}}\right)$ fits in a long exact sequence

$$
0 \longrightarrow G H^{1}\left(\widetilde{\mathbb{C}^{2}}\right) \longrightarrow G H^{1}(U) \oplus G H^{1}(V) \longrightarrow G H^{1}(U \cap V) \longrightarrow \cdots,
$$

so $G H^{1}\left(\widetilde{\mathbb{C}^{2}}\right)$ is a subspace of $G H^{1}(V)$. More precisely, $G H^{1}\left(\widetilde{\mathbb{C}^{2}}\right)$ corresponds to the holomorphic forms $d f\left(v_{2}\right)$, defined on a neighbourhood of the type change locus on $V$ which can be extended smoothly to $\widetilde{\mathbb{C}^{2}}$. But it is clear that any such form can be extended smoothly, just by multiplying $f$ by a bump function supported in a neighbourhood of the type change locus, hence $G H^{1}\left(\widetilde{C}^{2}\right)=G H^{1}(V)=\pi^{*} G H^{1}\left(\mathbb{C}^{2}\right)$.

Theorem 3.2. Let $M$ be a compact generalized complex manifold and $\widetilde{M}$ be the blow up of $M$ on a nondegenerate type change point. Then $G H^{i}(\widetilde{M})=\pi^{*} G H^{i}(M)$ for $i \neq 0$ and $G H^{0}(\widetilde{M})=\pi^{*} G H^{0}(M) \oplus \mathbb{C}$.

Proof. By Meyer-Vietoris, denoting by $\Sigma$ de exceptional divisor introduced by the blow up, we have the following map of exact sequences

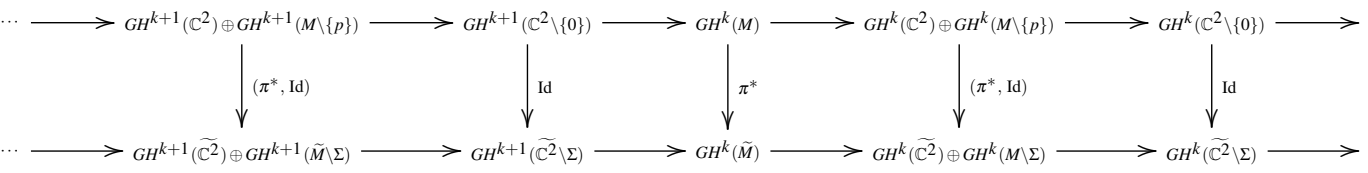


For $k=2$ and 1 the outer maps are isomorphisms (for $k=1$, this uses Lemma 3.1) and by the Five Lemma, the middle map is an isomorphism. For $k=0$ the sequence above yields $G H^{0}(\widetilde{M}) \cong G H^{0}\left(\widetilde{\mathbb{C}^{2}}\right) \oplus G H^{0}(M)$.

To compute the dimesion of $G H^{0}(M)$ we observe that blowing up increases the Euler characteristic by 1 , hence

$$
2 g b_{2}(\widetilde{M})-2 g b_{1}(\widetilde{M})+g b_{0}(\widetilde{M})=1+\chi(M)=1+2 g b_{2}(M)-2 g b_{1}(M)+g b_{0}(M),
$$

showing that $g b_{0}(\widetilde{M})=g b_{0}(M)+1$, i.e., $G H^{0}\left(\widetilde{\mathbb{C}^{2}}\right) \cong \mathbb{C}$.

The increase in the middle cohomology is related to the fact that the exceptional divisor introduced by the blow-up is a brane in $\widetilde{M}$ and these roughly correspond to classes in $G H^{0}(\widetilde{M})[1]$.

\section{A SURGERY}

In joint work with Gualtieri, the author introduced a surgery for generalized complex manifolds in [2] which is used to create type changing generalized complex manifolds out of ordinary symplectic manifolds. In this section we describe this surgery and compute the generalized cohomology of some manifolds obtained this way.

Given a 2-torus with trivial normal bundle embedded on $M^{4}$, a neighbourhood of this torus is diffeomorphic to $D^{2} \times T^{2}$. We can parametrize $D^{2} \backslash\{0\} \times T^{2}$ using polar coordinates $\left(r, \theta_{1}\right)$ for $D^{2} \backslash\{0\}$ and two angle coordinates $\left(\theta_{2}, \theta_{3}\right)$ for $T^{2}$. The surgery then consists in removing this neighbourhood of $T^{2}$ and gluing it back using the following diffeomorphism of $\partial\left(D^{2} \times T^{2}\right) \cong T^{3}$ :

$$
\varphi\left(\theta_{1}, \theta_{2}, \theta_{3}\right)=\left(\theta_{3}, \theta_{2},-\theta_{1}\right)
$$

This is a particular case of $\mathscr{C}^{\infty}$-log transforms. Observe that by construction $\hat{M} \backslash \hat{T} \cong$ $M \backslash T$, where $\hat{T}$ and $T$ represent the tori over $0 \in D^{2}$ in $\hat{M}$ and in $M$, respectively.

The result proved in [2] is

Theorem 4.1. If $M$ is symplectic and $T^{2} \hookrightarrow M^{4}$ is a symplectic torus with trivial normal bundle, then $\hat{M}$, the result of the surgery, has a type changing generalized complex structure integrable with respect to the 3-form Poincaré dual to the circle paramatrized by $\theta_{2}$.

This structure is obtained by identifying part of the symplectic locus of the generalized complex structure from Example 1.2 with the neighbourhood of $T^{2} \subset M^{4}$. This surgery was then used in [3] to prove that $(2 n+1) \mathbb{C} P^{2} \# \bar{m} \overline{\mathbb{C} P^{2}}$ has a generalized complex structure for all $n, m \in \mathbb{N}$. This was achieved by taking $M$ to be $E_{n}$, the symplectic fiber sum of $n$ copies of $\mathbb{C} P^{2} \# 9 \overline{\mathbb{C} P^{2}}$ and then performing the surgery in one of the regular fibers and following this process by blow-up/blow down of appropriate submanifolds. 
A common feature of all the examples in this family is that $H^{1}(\hat{M} \backslash \hat{T})=\{0\}$, where $\hat{T}$ is the type change torus. Now we shall prove that this implies that all the generalized cohomology of these manifolds concentrates in the middle dimension.

Theorem 4.2. Let $M^{4}$ be a simply connected, compact manifold and $\mathscr{J}$ be a type changing generalized complex structure whose type change locus $T$ is connected and nondegenerate. If $H^{1}(M \backslash T)=\{0\}$, then $G H^{2}(M)=G H^{1}(M)=\{0\}$ and $G H^{0}(M)=$ $\chi(M)$.

Proof. According to Lemma 2.1, $G H^{2}(M)=\{0\}$. Since the type change locus is compact and nondegenerate, it is a torus [2]. Letting $N$ be a small neighbourhood of the type change locus, according to Theorem $2.3 G H^{1}(N)$ is given by the pull back of holomorphic (1,0)-differentials $\alpha^{1,0}$ on the torus to the $N$. More precisely, the cohomology is generated by

$$
e^{B+i \omega} \alpha^{1,0}
$$

Since $H^{1}(M \backslash T)=0$, Mayer-Vietoris for the operator $\bar{\partial}_{\mathscr{J}}$ implies that $G H^{1}(M)$ can only be $\mathbb{C}$ or $\{0\}$ and what determines which is the case is whether the form (4.1) can be extended to the rest of $M$ as a $\bar{\partial} \mathscr{J}$-closed form, or equivalently, whether $\alpha^{1,0}$ can be extended as a closed form. Now, if $\alpha^{1,0} \neq 0$, it has nonvanishing integrals over both cycles in $H_{1}\left(T^{2}\right)$. Since $H^{1}(M \backslash T)=\{0\}$ both of these cycles are boundaries and hence $\alpha$ can not be extended as a closed form, therefore showing that $G H^{1}(M)=\{0\}$.

To finish the proof we recall that the Euler characteristic can be computed using the generalized cohomology, hence $G H^{0}(M)=\chi(M)$.

\section{5. $\partial \bar{\partial}$-LEMMA}

A generalized complex manifold satisfies the $\partial \bar{\partial}$-lemma if

$$
\operatorname{Im}(\partial) \cap \operatorname{ker}(\bar{\partial})=\operatorname{ker}(\partial) \cap \operatorname{Im}(\bar{\partial})=\operatorname{Im}(\partial \bar{\partial}) .
$$

In this short section we prove that the type changing generalized complex structure on $(2 n+1) \mathbb{C} P^{2} \# m \overline{\mathbb{C} P^{2}}$ encountered in [3] satisfies the $\partial \bar{\partial}$-lemma.

Theorem 5.1. If the generalized cohomology of a generalized complex manifold $M^{4}$ concentrates in $G H^{0}(M)$, then the generalized complex structure satisfies the $\partial \bar{\partial}$-lemma. In particular, the manifolds $(2 n+1) \mathbb{C} P^{2} \# m \overline{\mathbb{C}} P^{2}$ with the generalized complex structure described in the previous section satisfy the $\partial \bar{\partial}$-lemma.

Proof. We will prove this by checking that the lemma holds in every degree.

1) Let $\alpha_{2} \in \mathscr{U}^{2}$ be $\partial$ and $\bar{\partial}$-closed. Since there is no $\bar{\partial}$-cohomology is degree 2 , $\bar{\partial} \alpha_{2}=0$ implies that $\alpha_{2}=0$ and hence the lemma holds trivially.

2) Let $\alpha_{1} \in \mathscr{U}^{1}$ be $\partial$ and $\bar{\partial}$-closed. Again, since there is no cohomology in degree 1 , $\alpha_{1}=\bar{\partial} \beta_{2}$. Since there is no $\partial$-cohomology in degree 2 and $\partial \beta_{2} \in \mathscr{U}^{3}=\{0\}, \beta_{2}=\partial \beta_{1}$ and hence $\alpha_{1}=\bar{\partial} \partial \beta_{1}$.

3) Let $\alpha_{0} \in \mathscr{U}^{0}$ be $\partial$-closed and $\bar{\partial}$-exact, say $\alpha_{0}=\bar{\partial} \beta_{1}$. Then, from $\partial \bar{\partial} \beta_{1}=0$, we see that $\partial \beta_{1}$ is a $\bar{\partial}$-closed form in $\mathscr{U}^{2}$. Since $G H^{2}(M)=\{0\}$, we see that $\partial \beta_{1}$ must be 
$\bar{\partial}$-exact and hence must vanish, by Lemma 2.1 , showing that $\beta_{1}$ is $\partial$-exact, say $\beta_{1}=\partial \beta_{0}$ and $\alpha_{0}=-\partial \bar{\partial} \beta_{0}$.

The cases of $\alpha_{0} \in \mathscr{U}^{0} \partial$-exact and $\bar{\partial}$-closed as well as $\alpha_{-1} \in \mathscr{U}^{-1}$ and $\alpha_{-2} \in \mathscr{U}^{-2}$ follow by observing that $\bar{\alpha}$ falls in one of the three cases already studied, hence $\bar{\alpha}$ is in $\operatorname{Im}(\partial \bar{\partial})$, and hence so is $\alpha$.

\section{ACKNOWLEDGMENTS}

This research is supported by Nederlandse Organisatie voor Wetenschappelijk Onderzoek (NWO).

\section{REFERENCES}

1. G. R. CaVAlcanti. The decomposition of forms and cohomology of generalized complex manifolds. J. Geom. Phys., 57:121-132, 2007.

2. G. R. CAVAlcanti and M. Gualtieri. A surgery for generalized complex structures on 4manifolds. J. Differential Geom., 76:35-43, 2007.

3. G. R. CAVAlCAnti AND M. Gualtieri. Blow-up of generalized complex 4-manifolds. arXiv:0806.0872., 2008.

4. C. Chevalley. The algebraic theory of spinors and Clifford algebras. Springer-Verlag, Berlin, 1997. Collected works. Vol. 2, Edited and with a foreword by Pierre Cartier and Catherine Chevalley, With a postface by J.-P. Bourguignon.

5. M. CRAINIC. Generalized complex structures and lie brackets. 2004.

6. S. J. Gates, JR., C. M. Hull, AND M. RoČEK. Twisted multiplets and new supersymmetric nonlinear $\sigma$-models. Nuclear Phys. B, 248(1):157-186, 1984.

7. M. Gualtieri. Generalized Complex Geometry. D.Phil. thesis, Oxford University, 2003. math.DG/0401221.

8. N. Hitchin. Generalized Calabi-Yau manifolds. Q. J. Math., 54(3):281-308, 2003.

9. J.-L. Koszul. Crochet de Schouten-Nijenhuis et cohomologie. Astérisque, Numero Hors Serie:257-271, 1985. The mathematical heritage of Élie Cartan (Lyon, 1984).

10. U. Lindström, R. Minasian, A. Tomasiello, and M. ZabZine. Generalized complex manifolds and supersymmetry. Comm. Math. Phys., 257(1):235-256, 2005.

11. U. Lindstrom, M. RoceK, R. von UnGe, AND M. ZabZine. Generalized kahler manifolds and off-shell supersymmetry. Communications in Mathematical Physics, 269:833, 2007. 